\title{
High expression of miR-155 and miR-21 in the recurrence or metastasis of non-small cell lung cancer
}

\author{
SHUANG XU ${ }^{1}$ and LEI SHI ${ }^{2}$ \\ ${ }^{1}$ Department of Cardiothoracic Surgery, Daqing Oil Field General Hospital; ${ }^{2}$ Department of Hematology \\ and Oncology, Daqing Longnan Hospital, Daqing, Heilongjiang 163000, P.R. China
}

Received November 20, 2018; Accepted April 24, 2019

DOI: $10.3892 / \mathrm{ol} .2019 .10337$

\begin{abstract}
High expression of miR-155 and miR-21 in the recurrence or metastasis of non-small cell lung cancer (NSCLC) was investigated. Retrospective analysis on the clinical information of 180 patients with NSCLC was carried out. The patients were admitted to Daqing Oil Field General Hospital from February 2012 to March 2015 and they were the research group. Moreover, the physical examination information of 88 normal medical examinees were selected at the same period of time as the control group. In the research group, 68 patients diagnosed with NSCLC were the newly diagnosed group and 112 cases of recurrence or metastasis of NSCLC were the recurrence group. The quantitative real-time polymerase chain reaction was used to detect the expression levels of serum miR-115 and miR-21. In addition, the expression levels between miR-155 and miR-21 and the relationship between the recurrence rate and metastasis of NSCLC were analyzed. The impact on the prognosis of patients were also analyzed. The expression levels of serum miR-155 and miR-21 were higher in the research group than those in the control group $(\mathrm{P}<0.05)$. The expression levels of serum miR-155 and miR-21 were higher in the recurrence group than those in the newly diagnosed group $(\mathrm{P}<0.05)$. We followed up the patients in the research group for 36 months, the median survival time and mortality rate in the recurrence group was higher than that of in the newly diagnosed group $\left(\chi^{2}=9.705, \mathrm{P}<0.01\right)$. In conclusion, miR-155 and miR-21 were highly expressed in the serum of patients, however, the recurrence and metastasis of NSCLC were even higher. The highly expressed levels of miR-155 and miR-21 were associated with the recurrence and metastasis in NSCLC patients, affecting the prognosis of patients.
\end{abstract}

Correspondence to: Dr Shuang Xu, Department of Cardiothoracic Surgery, Daqing Oil Field General Hospital, 9 Zhongkang Street, Daqing, Heilongjiang 163000, P.R. China

E-mail: ers83p@163.com; 39883128@qq.com

Dr Lei Shi, Department of Hematology and Oncology, Daqing Longnan Hospital, 35 Aiguo Road, Daqing, Heilongjiang 163000, P.R. China

E-mail: 252392914@qq.com

Key words: miR-155, miR-21, non-small cell lung cancer, recurrence, metastasis, prognosis

\section{Introduction}

Lung cancer is the leading cause of cancer death globally, accounting for $18.20 \%$ of all cancer deaths. The incidence rate is ranked first in the global incidence of cancers, accounting for $12.70 \%$ of all cancer cases (1). According to statistics, among the number of lung cancer cases in Serbia of southeastern Europe, the incidence rates in men were higher than those of women and the mortality rates of male patients were four times higher than those of female patients. This may be due to the smoking habit in males (2). According to the histological type of lung cancer, it can be divided into small cell lung cancer and non-small cell lung cancer (NSCLC). NSCLC accounts for most causes of lung cancer, according to statistics, NSCLC accounts for $80 \%$ of all patients with lung cancers (3). NSCLC has no obvious symptoms in the early stage, normally patients are already in the advanced stage when obvious clinical symptoms or physiological reactions occur. Some statistics have shown that most NSCLC patients are already in the advanced stage at the time of diagnosis (4). According to the pathological type of NSCLC, it is mainly classified into large cell carcinoma, squamous cell carcinoma and adenocarcinoma. Among them, adenocarcinoma and squamous cell lung cancer are the main types of cancers (5). Surgical resection is the main treatment method for NSCLC, however, patients with NSCLC have a higher recurrence rate (6). With the development of medical technology, chemotherapy has become more effective in the treatment of NSCLC. Although the prognosis of patients with NSCLC has improved it is affected by drug resistance. Moreover, the five-year survival rate of NSCLC patients is still less than $20 \%$ and seriously affects people's health (7). Therefore, we have to continue to find effective tumor markers to judge the prognosis of NSCLC clinically.

microRNA (miRNA) is an endogenous non-proteinencoded small RNA, located in eukaryotic cells and approximately $22 \mathrm{nt}$ in length. Studies have shown that its expression has a certain impact on the occurrence, development, invasion, metastasis and prognosis of many malignant tumors, which has an important effect in inhibiting tumors or promoting tumor development (8). miRNA has a great significance in the diagnosis and treatment evaluation of NSCLC and can be used as a non-invasive biomarker for prognosis (9).

miR-21 is one of the miRNAs located on human chromosome $17 \mathrm{q} 23.2$ and with an abnormally high expression in many 
Table I. miR-155 and miR-21 and internal reference $\beta$-actin primer sequences.

\begin{tabular}{llc}
\hline Gene names & Upstream primer & Downstream primer \\
\hline$m i R-21$ & 5'-TGCGCTAGCTTATCAGACTGAT-3' & 5'-CCAGTGCAGGGTCCGAGGTATT-3' \\
$m i R$ - 155 & 5'-CTGTAT-CAAAAGGCCAACTGAA-3' & 5'-GTGTCTATCCT-TATGAATCGCCA-3' \\
$\beta$ - actin & 5'-AGCGGGAAATCGTGCGTGACA-3' & 5'-GTG-GACTTGGGAGAGGACTGG-3' \\
\hline
\end{tabular}

malignant tumors, also is a type of oncogene (10). Studies have shown that the growth and invasion of NSCLC cancer cells can be promoted by inhibiting the tumor suppressor phosphatase and tensin homolog deleted from chromosome 10 (PTEN). The expression levels of miR-21 were highly expressed in NSCLC patients and the expression of miR-21 was associated with TNM stages and distant metastasis in NSCLC patients (11). miR-155 is an important member of miRNA, the gene is located on chromosome 21 and it was processed by nonprotein-encoded transcripts of B cell fusion cluster genes (12). miR-155 promotes the growth and development of tumor cells by regulating a variety of cancer-promoting pathways and has a high expression in a variety of malignancies (13). Studies have shown that miR-155 was highly expressed in NSCLC and induces the proliferation and invasion of NSCLC cancer cells by directly inhibiting the programmed cell death gene 4 (14).

There are few studies on the high expression of miR-155 and miR-21 and the recurrence or metastasis of NSCLC patients. This research investigated the expression levels of serum miR-155 and miR-21 in patients with recurrence or metastasis of NSCLC. We also explored the relationship between the high expression of miR-155 and miR-21 and the recurrence or metastasis of NSCLC in order to provide clinical references.

\section{Materials and methods}

General information. Retrospective analysis of the clinical information of 180 patients with NSCLC admitted to Daqing Oil Field General Hospital (Daqing, China) from February 2012 to March 2015 were carried out. These patients were the research group. There were 124 cases of males and 56 cases of females, with an average age $66.37 \pm 7.21$ years. Physical examination information of 88 normal medical examinees was selected during the same period of time, and set as the control group. There were 63 cases of males and 25 cases of females, average age $64.98 \pm 7.23$ years. Sixty-eight patients in the research group were newly diagnosed with NSCLC, and were set as the newly diagnosed group and there were 46 cases of males and 22 cases of females. In addition, 112 patients with recurrence or metastasis of NSCLC were the recurrence group, there were 78 cases of males and 34 cases of females.

This investigation was approved by the Ethics Committee of Daqing Oil Field General Hospital, patients or their family members signed the informed consent form.

Inclusion and exclusion criteria. Inclusion criteria: Age $\geq 18$ years; no other hereditary diseases; patients with a complete clinical medical records or physical examination information; included were only patients who were treated in Daqing Oil Field General Hospital; no deaths occurred during the treatment and the patients have been discharged. Exclusion criteria: Patients with cardiovascular disease, patients with second primary tumors; patients who had taken antibiotics in the last three months; combined with severe liver and kidney dysfunction; combined with other connective tissue diseases, endocrine and metabolic diseases; and patients with a history of mental illness or family history of mental illness.

Main reagents and instruments. PCR amplifier (manufacturer: Tianjin Jinside Biotechnology Co., Ltd., Tianjin, China, model: ABI 2700); TRIzol extractant (manufacturer: Beijing Suo Laibao Technology Co., Ltd., Beijing, China, batch number: 15596-026); high-speed centrifuge (manufacturer: Anhui Zhongke Zhongjia Scientific Instrument Co., Ltd., Hefei, China, model: HC-2518R); reverse transcription RT kit (manufacturer: Shanghai Jiemei Gene Pharmaceutical Technology Co., Ltd., Shanghai, China, batch number: GMS20020.4); RT-qPCR kit (manufacturer: Shanghai Chuangye Biotechnology Co., Ltd., Shanghai, China, article number: ZY131042) were used in the present study. The miR-155 and miR-21 and the internal reference $\beta$-actin primer sequences were designed and produced by Suzhou Yuxun Biotechnology Co., Ltd. Suzhou, China (Table I).

Collection of serum. The operation steps were all carried out in a sterile environment, $6 \mathrm{ml}$ of the external elbow venous blood was extracted in the morning after all the experimental subjects had fasted for $8 \mathrm{~h}$. Blood was then placed in a vacuum blood collection tube for $25 \mathrm{~min}$, the serum was separated in a centrifuge at $3,000 \mathrm{x} \mathrm{g}$ at $22^{\circ} \mathrm{C}$ for approximately $15-20 \mathrm{~min}$. After separation, $350 \mu \mathrm{l}$ of the supernatant was placed in an EP tube. After being placed in liquid nitrogen for quick freezing, it was stored at $-80^{\circ} \mathrm{C}$.

Extraction of total RNA and synthesis of cDNA. The RNA was extracted using TRIzol, serum was taken from the refrigerator, $1.05 \mathrm{ml}$ TRIzol was added and agitated for $5 \mathrm{~min}$. The mixture was kept at room temperature for $10 \mathrm{~min}$ and $600 \mu \mathrm{l}$ of chloroform was added after the cells were completely lysed. The mixture was mixed until the solution was emulsified and milky white after which it was left to stand for $5 \mathrm{~min}$, following centrifugation at $800 \mathrm{xg}$ for $15 \mathrm{~min}$ at $4^{\circ} \mathrm{C}$. The top colorless supernatant was carefully absorbed into the centrifuge tube using a pipette, an equal volume of isopropanol was added and centrifuged for further $10 \mathrm{~min}$. After centrifugation, the precipitate was washed by adding $1 \mathrm{ml}$ of $75 \%$ ethanol to obtain RNA. The RNA integrity was tested by using $1 \%$ agarose gel electrophoresis. Then a qualified RNA sample was selected for cDNA synthesis, and reverse transcribed cDNA using $1 \mu \mathrm{l}$ of total RNA, the reaction was carried out under reaction conditions of $42^{\circ} \mathrm{C}$ for $55 \mathrm{~min}$. 
Table II. Comparison of baseline information between the research group and the control group [n (\%)] (mean \pm SD).

\begin{tabular}{|c|c|c|c|c|}
\hline Characteristics & Research group $(\mathrm{n}=180)$ & Control group $(\mathrm{n}=88)$ & $\chi^{2} / \mathrm{t}$ value & P-value \\
\hline Age (years) & $66.37 \pm 7.21$ & $64.98 \pm 7.23$ & 1.481 & 0.140 \\
\hline Sex & & & 0.205 & 0.674 \\
\hline Male & $124(68.89)$ & $63(71.59)$ & & \\
\hline Female & $56(31.11)$ & $25(28.41)$ & & \\
\hline $\operatorname{BMI}\left(\mathrm{kg} / \mathrm{m}^{2}\right)$ & & & 0.164 & 0.755 \\
\hline$<24$ & $141(78.33)$ & 67 (76.14) & & \\
\hline$\geq 24$ & $39(21.67)$ & $21(23.86)$ & & \\
\hline Smoking status & & & 1.636 & 0.240 \\
\hline Smoker & $136(75.56)$ & $60(68.18)$ & & \\
\hline Non-smoker & $44(24.44)$ & $28(31.82)$ & & \\
\hline Heart rate (times/min) & & & 0.642 & 0.503 \\
\hline$<60$ & $15(8.33)$ & $10(11.36)$ & & \\
\hline$\geq 60$ & $165(91.67)$ & $78(88.64)$ & & \\
\hline Fasting blood glucose $(\mathrm{mmol} / \mathrm{l})$ & $4.48 \pm 0.62$ & $4.53 \pm 0.56$ & 0.640 & 0.523 \\
\hline $\mathrm{Hb}(\mathrm{g} / \mathrm{l})$ & $115.24 \pm 14.26$ & $117.25 \pm 13.63$ & 1.099 & 0.273 \\
\hline $\mathrm{RBC}\left(\mathrm{x} 10^{12} / \mathrm{l}\right)$ & $4.51 \pm 0.26$ & $4.55 \pm 0.35$ & 1.051 & 0.294 \\
\hline PLT $\left(x 10^{9} / 1\right)$ & $224.24 \pm 36.73$ & $218.99 \pm 55.36$ & 0.924 & 0.357 \\
\hline
\end{tabular}

Detection of the expression levels of miR-155 and miR-21. The expression levels of miR-155 and miR-21 were detected by RT-qPCR and the reaction system of $20 \mu \mathrm{l}$ volume. First, pre-denaturation at $95^{\circ} \mathrm{C}$ for $15 \mathrm{~min}$, then denaturation at $94^{\circ} \mathrm{C}$ for $15 \mathrm{sec}$, annealing at $56^{\circ} \mathrm{C}$ for $30 \mathrm{sec}$ and extension at $70^{\circ} \mathrm{C}$ for $30 \mathrm{sec}$, and repetition of the 40 cycles. The experiment was repeated 3 times and completed within 30 days. The standard curve was based on the standard concentration and the average value of the cycle depreciation corresponding to each concentration. According to the standard curve, the instrument automatically calculates the copy number $/ \mathrm{ml}$ of miR-155, miR-21 and $\beta$-actin in serum. In addition, the ratio of miR-155, miR-21 and $\beta$-actin was used as the expression level of miR-155 and miR-21.

Follow up for the research group. After the treatment, all NSCLC patients discharged from the hospital were followed up once every month for 36 months, mainly by telephone. Home visit survey or consultation with relatives were conducted for patients who had poor telephone follow-ups and elderly patients or those with poor physical fitness. This ensured the accurate recording of deaths in the research group.

Statistical analysis. Statistical analysis was performed using SPSS19.0 [Yiyun (Shanghai) Information Technology Co., Ltd., Shanghai, China] and measurement data was expressed as mean \pm standard deviation (mean $\pm \mathrm{SD}$ ). The t-test was used to compare the measurement data between all the groups and the Chi-square test was used to compare the countable data between the groups. Survival analysis was performed using Kaplan-Meier and the log-rank test. $\mathrm{P}<0.05$ was considered to indicate a statistically significant difference.

\section{Results}

General baseline information. Factors including age, sex, body mass index, heart rate, smoking status, fasting blood glucose level, hemoglobin $(\mathrm{Hb})$ value, red blood cell $(\mathrm{RBC})$ value, and platelet (PLT) value between the research and control groups were compared. The difference between the groups was not statistically significant $(\mathrm{P}>0.05)$ (Table II).

Expression levels of serum miR-155 and miR-21 between the research and control groups. The experiment results showed that the expression level of miR-21 in the research group was $7.13 \pm 3.76$, which was higher than that in the control group $(1.45 \pm 0.62)$ and the difference between two groups was statistically significant $(\mathrm{t}=14.060, \mathrm{P}<0.01)$. The expression level of miR-155 in the research group was $6.34 \pm 3.93$, which was higher than that in the control group $(1.32 \pm 0.52)$. The difference between two groups was statistically significant $(\mathrm{t}=11.920, \mathrm{P}<0.01)$ (Fig. 1).

Clinical information in the subgroup of the research group. Factors including age, sex, body mass index, smoking status, tumor diameter, differentiation, histological type, and TNM stage between the recurrence group and the newly diagnosed group were compared. The difference between the groups was not statistically significant $(\mathrm{P}>0.05)$ (Table III).

Expression of serum miR-155 and miR-21 in the subgroup of the research group. The results of the experiment showed that the expression level of miR-21 in the recurrence group was $8.18 \pm 2.71$, which was higher than that in the newly diagnosed group $(5.33 \pm 1.96)$. The difference between the groups was statistically significant $(\mathrm{t}=7.552, \mathrm{P}<0.01)$. The expression level 
Table III. Comparison of clinical information between the recurrence group and the newly diagnosed group [n (\%)].

\begin{tabular}{|c|c|c|c|c|}
\hline Characteristics & Recurrence group $(\mathrm{n}=112)$ & Newly diagnosed group $(\mathrm{n}=68)$ & $\chi^{2}$ value & P-value \\
\hline Age (years) & & & 0.342 & 0.631 \\
\hline$<65$ & $38(33.93)$ & $26(38.24)$ & & \\
\hline$\geq 65$ & $74(66.07)$ & $42(61.76)$ & & \\
\hline Sex & & & 0.079 & 0.868 \\
\hline Male & $78(69.64)$ & $46(67.65)$ & & \\
\hline Female & $34(30.36)$ & $22(32.35)$ & & \\
\hline $\operatorname{BMI}\left(\mathrm{kg} / \mathrm{m}^{2}\right)$ & & & 0.418 & 0.579 \\
\hline$<24$ & 86 (76.79) & $55(80.88)$ & & \\
\hline$\geq 24$ & $26(23.21)$ & $13(19.12)$ & & \\
\hline Smoking status & & & 0.880 & 0.377 \\
\hline Smoker & $82(73.21)$ & $54(79.41)$ & & \\
\hline Non-smoker & $30(26.79)$ & $14(20.59)$ & & \\
\hline Tumor diameter & & & 0.116 & 0.752 \\
\hline$<5 \mathrm{~cm}$ & $40(35.71)$ & $26(38.24)$ & & \\
\hline$\geq 5 \mathrm{~cm}$ & $72(64.29)$ & $42(61.76)$ & & \\
\hline Histological type & & & 0.095 & 0.878 \\
\hline Squamous cell carcinoma & $57(50.89)$ & $33(48.53)$ & & \\
\hline Adenocarcinoma & $55(49.11)$ & $35(51.47)$ & & \\
\hline Differentiation & & & 0.702 & 0.482 \\
\hline Mid and low & $81(72.32)$ & $53(77.94)$ & & \\
\hline High & $31(27.68)$ & $15(22.06)$ & & \\
\hline TNM stages & & & 0.142 & 0.755 \\
\hline I-II & $46(41.07)$ & $26(38.24)$ & & \\
\hline III-IV & $66(58.93)$ & $42(61.76)$ & & \\
\hline
\end{tabular}

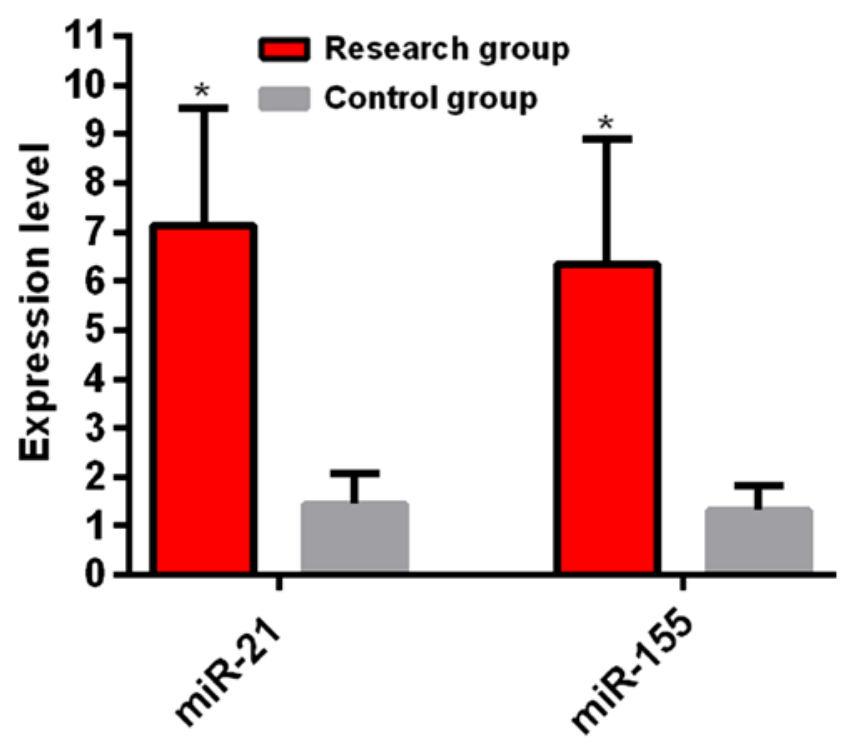

Figure 1. Comparison of serum miR-155 and miR-21 expression levels between the research group and the control group. RT-qPCR results showed that the expression levels of miR-21 and miR-155 in the research group were higher than those in the control group. ${ }^{*} \mathrm{P}<0.05$, the difference was statistically significant compared with the control group.

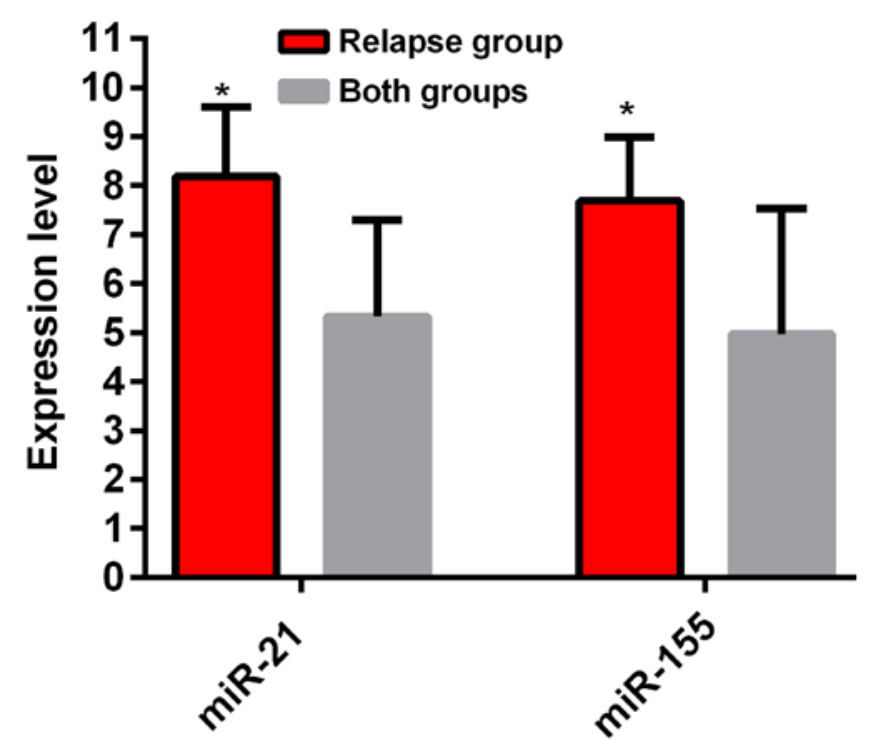

Figure 2. Comparison of serum miR-155 and miR-21 expression levels between the recurrence group and the newly diagnosed groups. RT-qPCR results showed that the expression levels of miR-21 and miR-155 in the recurrence group were higher than those in the newly diagnosed group. ${ }^{*} \mathrm{P}<0.05$, the difference was statistically significant compared with the control group.

The difference between the groups was statistically significant $(\mathrm{P}<0.05)$ (Fig. 2). 


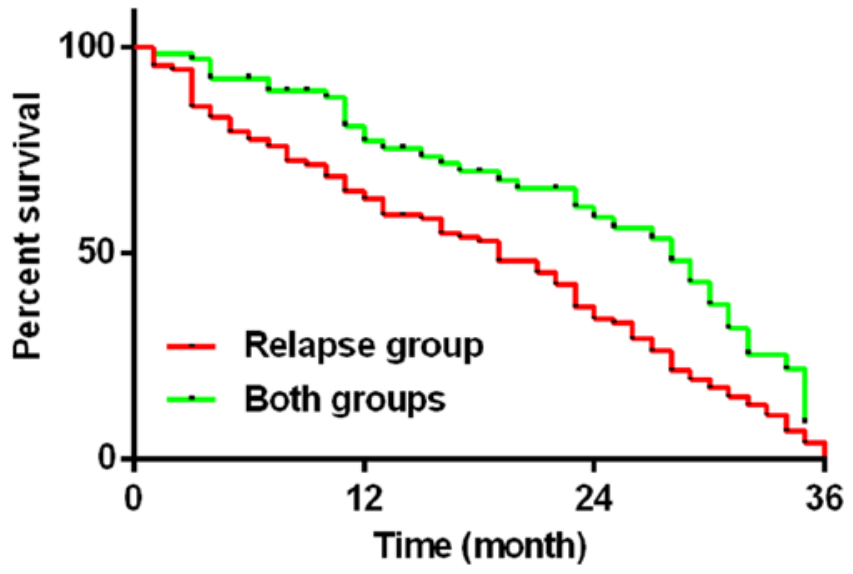

Figure 3. Comparison of death rate between the recurrence group and the newly diagnosed group. Kaplan-Meier test results showed that the median survival time in the recurrent group was 19 months. The survival time in the newly diagnosed group was 28 months and the difference between the groups was statistically significant $\left(\chi^{2}=9.705, \mathrm{P}<0.01\right)$. During the follow-up period, the mortality rate in the recurrence group was $91.96 \%$ and the newly diagnosed group was $57.35 \%$. The difference between the two groups was statistically significant $\left(\chi^{2}=9.705, \mathrm{P}<0.01\right)$

Subgroup of the research group. A 36-month fllow-up was conducted to both the recurrence group and the newly diagnosed group. At follow-up, 103 cases of patients died in the recurrence group, with a median survival time of 19 months and a mortality rate of $91.96 \%$. In addition, 39 patients died in the newly diagnosed group, the median survival time was 28 months and the mortality rate was $57.35 \%$. The difference between two groups was statistically significant $\left(\chi^{2}=9.705\right.$, $\mathrm{P}<0.01$ ) (Fig. 3).

\section{Discussion}

Lung cancer is the most malignant tumor with the highest morbidity and mortality rate in the world (15). Assessment of the prognosis of NSCLC is currently carried out using tissue cytological examination. However, these are intrusive inspection methods and often require obtaining tissues or cells, via lung puncture, biopsy or bronchoscopy. These methods are not readily accepted by patients due to inconvenience and trauma (16). In addition, a non-invasive, easy-to-use and highly accurate biomarker is required to assess the prognosis of NSCLC clinically.

miR-21 and miR-155 both have an important effect in the occurrence and development of lung cancer. Studies have shown that miR-155 has considerable regulatory effect in the proliferation, differentiation and growth of T cells (17). miR-21 causes signal transduction and activation on transcriptional activator 3 , which increases the vascular endothelial growth factor levels in recipient cells. This indirectly leads to malignant transformation of human bronchial epithelioid cells (18). The expression levels of miR-21 and miR-155 are elevated in various solid malignant tumors such as breast cancer, B cell lymphoma, lung cancer and colon cancer. Moreover, decreased expression levels of miR-21 and miR-155 can induce apoptosis and inhibit the proliferation and invasion of cells (19).

Xue et al (20) reported that the expression levels of miR-21 and miR-155 are increased in patients with NSCLC. In addition, silencing miR-21 and miR-155 can effectively inhibit the progression of NSCLC in the animals of xenotransplantation. Furthermore, combined inhibition of miR-21 and miR-155 can effectively improve the therapeutic effect of NSCLC, which is similar to our research results. We studied the expression levels of miR-21 and miR-155 in the serum between normal and NSCLC patients. It was found that miR-21 and miR-155 were highly expressed in the serum of NSCLC patients, which were higher than those in the normal patients $(\mathrm{P}<0.05)$. We further investigated the expression levels of miR-21 and miR-155 of the serum in patients with recurrence of NSCLC or metastasis and found that the expression levels of miR-21 and miR-155 with recurrence or metastatic NSCLC were higher than those in the newly diagnosed patients with NSCLC $(\mathrm{P}<0.05)$. The results of Yang et al (21) were similar to our results, they found that the high expression levels of miR-21 and miR-155 in the post-operative recurrence and metastasis of patients with NSCLC were higher than those of patients with low expression levels. However, the survival rate was lower than that of patients with a low expression level. We conducted a 36-month follow up in the newly diagnosed patients and NSCLC patients with recurrence or metastasis. The mortality rate of the NSCLC with recurrence or metastasis was $91.96 \%$, the median survival time was 19 months and the initial diagnosis of NSCLC patients had a mortality rate of $57.35 \%$ and the median survival time was 28 months. The difference in survival rate between the two groups was statistically significant $(\mathrm{P}<0.05)$. Moreover, patients with recurrence or metastasis of NSCLC had a poor survival rate. The results of this investigation are similar to those of Lashiotaki et al (22) who considered that the high expression of miR-21 and miR-155 may reduce the overall survival and recurrence-free survival rate in patients with NSCLC. This can lead to poor prognosis in patients with NSCLC and reduces patient survival time. Donnem et al (23) reported that highly expressed miR-155 has a significant negative impact on the survival rates and prognosis of NSCLC and it is an independent prognostic factor for NSCLC. Gironella et al (24) considered that miR-155 is abnormally highly expressed in pancreatic cancer. It may be caused by inhibition of the mRNA function of TP53INP1 and resulted in a decrease in its expression level. Therefore, it inhibits tumor cell apoptosis and promotes tumor cell proliferation. In addition, Meng et al (25) believe that the expression level of PTEN can be increased by inhibiting the expression of miR-21 in liver cancer cells and therefore the proliferation and metastasis of tumors were inhibited. The PTEN gene may be impacted by miR-21 and could affect the metastasis and recurrence in tumors. This indicates that high expression of miR-21 and miR-155 is associated with recurrence and metastasis of tumors.

Retrospective case analysis was used during this study and the pathology was strictly selected according to the inclusion and exclusion criteria. The basic patient information investigation, clinical examination, and special inspection were strictly recorded and organized. Moreover, a 36-month follow-up was conducted in the NSCLC patients, this accurately recorded the patient death rate and obtained a reliable result.

miR-21 and miR-155 were highly expressed in the serum of patients with NSCLC, and the expression levels of serum in NSCLC patients with recurrence and metastasis was even higher. Highly expressed miR-21 and miR-155 were associated 
with recurrence and metastasis in patients with NSCLC reducing survival rate and affecting prognosis of patients.

\section{Acknowledgements}

Not applicable.

\section{Funding}

No funding was received.

\section{Availability of data and materials}

The datasets used and/or analyzed during the present study are available from the corresponding author on reasonable request.

\section{Authors' contributions}

SX drafted the manuscript and was responsible for the extraction of total RNA and synthesis of cDNA. SX and LS performed PCR. Both authors read and approved the final manuscript.

\section{Ethics approval and consent to participate}

The study was approved by the Ethics Committee of Daqing Oil Field General Hospital (Daqing, China). Signed informed consents were obtained from the patients or the guardians.

\section{Patient consent for publication}

Not applicable.

\section{Competing interests}

The authors declare that they have no competing interests.

\section{References}

1. Scagliotti GV, Szczesna A, Ramlau R, Cardenal F, Mattson K, Van Zandwijk N, Price A, Lebeau B, Debus J and Manegold C: Docetaxel-based induction therapy prior to radiotherapy with or without docetaxel for non-small-cell lung cancer. Br J Cancer 94: 1375-1382, 2006.

2. Kocić B, Petrović B, Rancić N and Ilić M: Lung cancer trends in Southeastern Serbia. Cent Eur J Public Health 21: 17-21, 2013.

3. Meza R, Meernik C, Jeon J and Cote ML: Lung cancer incidence trends by gender, race and histology in the United States, 1973-2010. PLoS One 10: e0121323, 2015.

4. Pathak AK, Bhutani M, Mohan A, Guleria R, Bal S and Kochupillai V: Non small cell lung cancer (NSCLC): Current status and future prospects. Indian J Chest Dis Allied Sci 46 191-203, 2004

5. Haroun RA, Zakhary NI, Mohamed MR, Abdelrahman AM, Kandil EI and Shalaby KA: Assessment of the prognostic value of methylation status and expression levels of FHIT, GSTP1 and p16 in non-small cell lung cancer in Egyptian patients. Asian Pac J Cancer Prev 15: 4281-4287, 2014.

6. Kuo CH, Wu CY, Lee KY, Lin SM, Chung FT, Lo YL, Liu CY, Hsiung TC, Yang CT and Wu YC: Chronic obstructive pulmonary disease in stage I non-small cell lung cancer that underwent anatomic resection: The role of a recurrence promoter. COPD 11: 407-413, 2014.

7. Kumpiro S, Sriuranpong V and Areepium N: Impact of the copper transporter protein 1 (CTR1) polymorphism on adverse events among advanced nonsmall cell lung cancer patients treated with a carboplatin/gemcitabine regimen. Asian Pac J Cancer Prev 17: 4391-4394, 2016.
8. Merino MJ, Gil S, Macias CG and Lara K: The unknown microRNA expression of male breast cancer. Similarities and differences with female ductal carcinoma. Their role as tumor biomarker. J Cancer 9: 450-459, 2018.

9. Feng B, Zhang K, Wang R and Chen L: Non-small-cell lung cancer and miRNAs: Novel biomarkers and promising tools for treatment. Clin Sci (Lond) 128: 619-634, 2015.

10. Ribas J, Ni X, Castanares M, Liu MM, Esopi D, Yegnasubramanian S, Rodriguez R, Mendell JT and Lupold SE: A novel source for miR-21 expression through the alternative polyadenylation of VMP1 gene transcripts. Nucleic Acids Res 40: 6821-6833, 2012.

11. Zhang JG, Wang JJ, Zhao F, Liu Q, Jiang K and Yang GH: MicroRNA-21 (miR-21) represses tumor suppressor PTEN and promotes growth and invasion in non-small cell lung cancer (NSCLC). Clin Chim Acta 411: 846-852, 2010.

12. Xie Q, Chen X, Lu F, Zhang T, Hao M, Wang Y, Zhao J, McCrae MA and Zhuang $\mathrm{H}$ : Aberrant expression of microRNA 155 may accelerate cell proliferation by targeting sex-determining region $\mathrm{Y}$ box 6 in hepatocellular carcinoma. Cancer 118: 2431-2442, 2012.

13. Gao Y, Fu S, Jiang W, Li B, Tian Y and Fu X: Association of MiR-155 expression with prognosis in resected stage III non-small cell lung cancer. Zhongguo Fei Ai Za Zhi 17: 417-423, 2014 (In Chinese).

14. Liu F, Song D, Wu Y, Liu X, Zhu J and Tang Y: MiR-155 inhibits proliferation and invasion by directly targeting PDCD4 in non-small cell lung cancer. Thorac Cancer 8: 613-619, 2017.

15. Gyoba J, Shan S, Roa W and Bédard EL: Diagnosing lung cancers through examination of micro-RNA biomarkers in blood, plasma, serum and sputum: A review and summary of current literature. Int J Mol Sci 17: 494, 2016.

16. Roesel C, Terjung S, Weinreich G, Hager T, Chalvatzoulis E, Metzenmacher M and Welter S: Sarcomatoid carcinoma of the lung: A rare histological subtype of non-small cell lung cancer with a poor prognosis even at earlier tumour stages. Interact Cardiovasc Thorac Surg 24: 407-413, 2017.

17. Liu J, Shi K, Chen M, Xu L, Hong J, Hu B, Yang X and Sun R: Elevated miR-155 expression induces immunosuppression via CD39(+) regulatory T-cells in sepsis patient. Int J Infect Dis 40: 135-141, 2015.

18. Liu Y, Luo F, Wang B, Li H, Xu Y, Liu X, Shi L, Lu X, Xu W, Lu L, et al: STAT3-regulated exosomal miR-21 promotes angiogenesis and is involved in neoplastic processes of transformed human bronchial epithelial cells. Cancer Lett 370: 125-135, 2016.

19. Wang Y, Li J, Tong L, Zhang J, Zhai A, Xu K, Wei L and Chu M: The prognostic value of miR-21 and miR-155 in non-small-cell lung cancer: A meta-analysis. Jpn J Clin Oncol 43: 813-820, 2013.

20. Xue X, Liu Y, Wang Y, Meng M, Wang K, Zang X, Zhao S, Sun X, Cui L, Pan L, et al: MiR-21 and MiR-155 promote non-small cell lung cancer progression by downregulating SOCS1, SOCS6, and PTEN. Oncotarget 7: 84508-84519, 2016.

21. Yang M, Shen H, Qiu C, Ni Y, Wang L, Dong W, Liao Y and Du J: High expression of miR-21 and miR-155 predicts recurrence and unfavourable survival in non-small cell lung cancer. Eur J Cancer 49: 604-615, 2013.

22. Lasithiotaki I, Tsitoura E, Koutsopoulos A, Lagoudaki E, Koutoulaki C, Pitsidianakis G, Spandidos DA, Siafakas NM, Sourvinos G and Antoniou KM: Aberrant expression of miR-21, miR-376c and miR-145 and their target host genes in Merkel cell polyomavirus-positive non-small cell lung cancer. Oncotarget 8: 112371-112383, 2016.

23. Donnem T, Eklo K, Berg T, Sorbye SW, Lonvik K, Al-Saad S, Al-Shibli K, Andersen S, Stenvold H, Bremnes RM, et al: Prognostic impact of MiR-155 in non-small cell lung cancer evaluated by in situ hybridization. J Transl Med 9: 6, 2011.

24. Gironella M, Seux M, Xie MJ, Cano C, Tomasini R, Gommeaux J, Garcia S, Nowak J, Yeung ML, Jeang KT, et al: Tumor protein 53 -induced nuclear protein 1 expression is repressed by miR-155, and its restoration inhibits pancreatic tumor development. Proc Natl Acad Sci USA 104: 16170-16175, 2007.

25. Meng F, Henson R, Wehbe-Janek H, Ghoshal K, Jacob ST and Patel T: MicroRNA-21 regulates expression of the PTEN tumor suppressor gene in human hepatocellular cancer. Gastroenterology 133: 647-658, 2007.

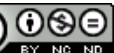

This work is licensed under a Creative Commons Attribution-NonCommercial-NoDerivatives 4.0 International (CC BY-NC-ND 4.0) License. 